\title{
La interpretación del fenómeno de las mareas como foco para el diseño de una propuesta didáctica
}

\author{
María Armario Bernal (iD, Natalia Jiménez-Tenrio iD, José María Oliva iD \\ Área de Didáctica de las Ciencias Experimentales. Departamento de Didáctica. Universidad de Cádiz. \\ España.maria.armario@uca.es,natalia.jimenez@uca.es, josemaria.oliva@uca.es
}

[Recibido: 26 marzo 2021. Revisado: 2 junio 2021. Aceptado: 5 agosto 2021]

\begin{abstract}
Resumen: Se presenta una secuencia didáctica diseñada para facilitar la comprensión del fenómeno de las mareas e involucrar a los participantes en procesos de modelización. Esto implica la construcción, revisión y reconstrucción de modelos, así como la reflexión en torno a su naturaleza y su papel en la enseñanza de la ciencia. El objetivo de este artículo es describir y caracterizar dicha secuencia, así como su implementación en el aula. Ésta sigue las pautas marcadas en las investigaciones de diseño: etapa preparatoria, etapa de implementación de dos ciclos y estudio retrospectivo. Los resultados del estudio preparatorio reafirmaron las dificultades en la enseñanza y aprendizaje de este tema: los profesores en las entrevistas mostraron su complejidad en diferentes ámbitos; el análisis de los libros de texto detectó posibles mejoras escritas y visuales, y los cuestionarios previos identificaron en los estudiantes ideas iniciales alejadas del modelo de ciencia escolar. En cuanto a la implementación, la trayectoria de progresión propuesta resultó ser apropiada, al igual que la organización de las actividades en el marco de la modelización. Además, se obtuvieron mejoras en los resultados del segundo ciclo de implementación. Por último, el análisis retrospectivo conllevó la realización de dos grandes tipos de cambios en la secuencia inicial y detectó una progresión en los modelos de la mayoría de los estudiantes.
\end{abstract}

Palabras clave: Estudio retrospectivo; Investigación basada en el diseño; Mareas; Modelos; Secuencia de enseñanza-aprendizaje.

\section{The interpretation of the tides phenomenon as a focus for the design of a didactic proposal}

\begin{abstract}
A didactic sequence designed to facilitate the understanding of the tides phenomenon and involve participants in modeling processes is presented. This involves the construction, revision and reconstruction of models, as well as reflection on their nature and their role in the teaching of science. The objective of this article is to describe and characterize said sequence, as well as its implementation in the classroom. This one follows the guidelines set by design based research: preparatory stage, two-cycle implantation stage, and retrospective study. The results of the preparatory study reaffirmed the difficulties in teaching and learning this topic: the teachers in the interviews showed its complexity in different areas, the analysis of the textbooks detected possible written and visual improvements, and the previous questionnaires identified in the students' initial ideas were far removed from the school science model. Regarding implementation, the proposed progression trajectory turned out to be appropriate, as was the organization of activities within the framework of modeling. In addition, improvements were obtained in the results of the second implementation cycle. Finally, the retrospective analysis involved the realization of two main types of changes in the initial sequence and detected a progression in the models of most of the students.
\end{abstract}

Keywords: Retrospective study; Design based research; Tides; Models; Teaching-learning sequences.

Para citar este artículo: Armario, M., Jiménez-Tenorio, N., y Oliva. J.M. (2021) La interpretación del fenómeno de las mareas como foco para el diseño de una propuesta didáctica en formación inicial de maestros. Revista Eureka sobre Enseñanza y Divulgación de las Ciencias 18(3), 3802 .doi: 10.25267/Rev_Eureka_ensen_divulg_cienc.2021.v18.i3.3802

\section{Introducción}

La formación inicial de maestros en didáctica de las ciencias resulta una labor compleja. Primero, porque su formación previa en contenidos científicos es limitada y diversa, coexistiendo estudiantes que abandonaron el estudio de las ciencias a los 14-15 años ( $\left.3^{\circ} \mathrm{ESO}\right)$, con una minoría que estudiaron bachillerato de ciencias y algunos que provienen de titulaciones de formación profesional. Segundo, porque la proporción de créditos que los 
títulos de maestros reservan a la formación en contenidos científicos y su didáctica es escasa, haciendo complicado compatibilizar ambas facetas.

Desde hace años, en la Universidad de Cádiz, intentamos afrontar esta situación implementando una educación científica vivencial, de manera que los docentes experimenten aquello que sería deseable que luego aplicasen con sus alumnos. De este modo, se consigue una coherencia entre el medio y el mensaje y una vía para optimizar el poco tiempo disponible.

En este marco, se considera que la enseñanza basada en modelización es una forma interesante de canalizar este planteamiento, haciendo partícipe a los futuros maestros de los procesos de construcción, uso, revisión y cambio de modelos (Gilbert y Justi 2016), dentro de un contexto de trabajo reflexivo y colaborativo, que parte de sus modelos personales que evolucionan hacia modelos más sofisticados (Clement 2000). En este proceso, los alumnos tienen la oportunidad de aportar ideas, tomar decisiones y avanzar en su conocimiento, monitorizados por el profesor, implicándose en prácticas científicas y en el desarrollo de su competencia de modelización (Nicolaou y Constantinou 2014).

Dado que los temas relacionados con el sistema Sol-Tierra-Luna (S-T-L) han sido emblemáticos como contenidos curriculares para involucrar a maestros en formación en procesos de modelización, nosotros también los hemos elegido como eje de nuestro trabajo. De hecho, existen numerosas publicaciones en las que el foco se sitúa en la formación de maestros en temáticas relacionadas con el ciclo día-noche o el fenómeno de las estaciones. En nuestro caso, lo abordamos a través del estudio del fenómeno de las mareas, por cuanto es un tema que ha sido mucho menos investigado y que, sin embargo, forma parte del día a día del alumnado de una facultad, como la nuestra, situada a escasos metros del mar.

Este artículo tiene por finalidad caracterizar una Secuencia de Enseñanza-Aprendizaje (SEA), cuyo objetivo era que los estudiantes construyeran un modelo para el fenómeno de las mareas como aplicación de otro más general abordado con anterioridad en torno al sistema S-T-L. Aunque el fenómeno de las mareas era el objeto central del estudio de diseño, la construcción y evaluación de modelos en este ámbito constituiría también una ocasión para que los estudiantes profundizasen en una comprensión más general sobre el sistema S-T-L.

Su implementación se desarrolló a lo largo de dos años consecutivos, de modo que la evaluación de resultados durante el primer ciclo de implementación sirvió para modificar la secuencia y volver a implementarla en el curso posterior con otro grupo de estudiantes del mismo nivel.

\section{El fenómeno de las mareas en la ciencia escolar}

El fenómeno de las mareas resulta un tópico árduo, ya que su comprensión requiere la construcción de un modelo que encierra complejidad y abstracción (Galili y Lehavi 2003; Hartel 2000; Norsen, Dreese y West 2017; Railsback 1991). Por ello, y al objeto de llevar a cabo la transposición didáctica, se puede considerar un modelo simplificado en el que la Tierra no tiene continentes y está cubierta por una hidrosfera que la envuelve. La fuerza gravitacional ejercida por la Luna, y en menor medida por el Sol, deforma el planeta y sus océanos, dando como resultado una masa de agua (con la Tierra en su interior) de forma elipsoide con dos "protuberancias", una a cada lado de la Tierra. Esta deformación se debe a la diferencia de fuerzas generada entre los puntos de la superficie del planeta más cercanos y más distantes con respecto a la Luna (Figura 1). Estas fuerzas atractivas no llegan a provocar un acercamiento progresivo entre la Tierra y la Luna, debido a que, en realidad, ambos astros no están estáticos, 
sino que se trasladan orbitalmente en torno al centro de masas del sistema Tierra-Luna (T-L) ${ }^{1}$ (Simanek 2015).
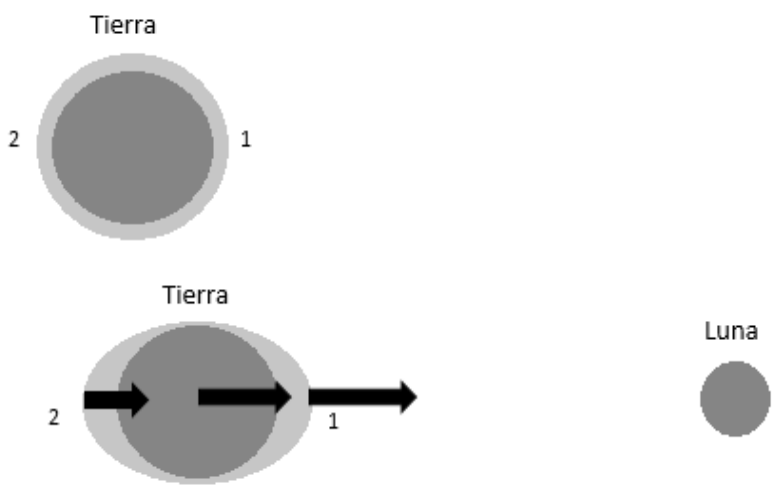

Figura 1. Generación de las protuberancias de las mareas a través de la fuerza gravitatoria de la Luna (Armario, Jiménez-Tenorio y Oliva 2019)

La deformación resultante provoca ciclos de mareas de aproximadamente $12 \mathrm{~h}$, ya que la Tierra gira sobre su eje una vez cada $24 \mathrm{~h}$. Esto implica la existencia de dos ciclos mareales por día y que, en puntos de la misma latitud situados en lados opuestos del globo (es decir, separados por $180^{\circ}$ de longitud), la altura y el sentido del cambio de la marea sean el mismo.

Se trata de una representación estática y muy simplificada, ya que la rotación del sistema T-L en su conjunto genera un efecto de arrastre sobre las dos protuberancias, con el resultado de que ambas no quedan alineadas según la línea imaginaria que une la Tierra con la Luna. Además, la existencia de los continentes impide el desplazamiento lateral uniforme del agua de los océanos hacia los dos lados del planeta. Por tanto, el patrón global de mareas es el resultado de muchos factores, incluida la profundidad y la forma de las cuencas oceánicas, la fricción producida por el fondo marino irregular y la topografía de las costas (Simanek 2015). De hecho, debido a estos factores, hay lugares en el planeta que experimentan solo un ciclo de mareas por día.

Sin embargo, trabajar con un modelo científico tan complejo va más allá de los objetivos de este estudio y de nuestros propósitos como formadores de maestros. Por ello, recurrimos a una versión simplificada del modelo científico como la esbozada anteriormente para nuestro diseño, que permite una primera comprensión del fenómeno y la realización de predicciones.

La bibliografía sobre la comprensión del fenómeno en los estudiantes sugiere la existencia de representaciones mentales muy alejadas de una explicación como ésta. Por ejemplo, Ballantyne (2004) detectó que los niños de 10 a 11 años tendían a explicar las mareas en términos de la acción del viento, las corrientes o las olas. Viiri (2000) estudió las explicaciones de estudiantes y profesores de secundaria finlandeses en formación inicial, identificando distintas categorías de respuestas, la mayoría muy distantes a las deseables. Una parte adoptaba un modelo local o topocéntrico ${ }^{2}$ situando el observador en la propia orilla y atribuyendo las causas a factores como la lluvia. Otra parte, la mayoría, concebía las mareas como resultado de un efecto de arrastre de toda la masa oceánica hacia la parte del globo más cercana a la Luna, consecuencia

\footnotetext{
$\overline{{ }^{1} \text { Ese movimiento orbital del sistema, }}$, como un todo, solo puede entenderse teniendo en cuenta otras fuerzas adicionales, ya que no debemos olvidar que, además de las fuerzas indicadas en la figura 1, sobre los puntos 1 y 2 actúan además fuerzas internas con el planeta sólido. De hecho, la fuerza neta que actúa sobre 2 debe ser mayor que sobre 1, dado que la fuerza centrípeta es mayor a medida que aumenta el radio de la trayectoria.

2 Entendido como un modelo topocéntrico desarrollado desde la visión de un observador ubicado en un punto determinado de la superficie terrestre (Galperin y Raviolo 2014).
} 
de la atracción gravitatoria. De este modo la gravedad, que para ellos procede sólo de la Luna, tendería a hacer que las aguas de los océanos se acumulen en un lado de la Tierra y retrocedan en el otro, lo que implicaría un modelo con un solo abultamiento y, en consecuencia, un solo ciclo de mareas al día. Sin embargo, muy pocos fueron capaces de hacer una descripción adecuada compatible con un ciclo mareal cada $12 \mathrm{~h}$, y casi ninguno usó una explicación conforme a la existencia de un diferencial de fuerzas, como requiere una comprensión adecuada del fenómeno. Resultados muy similares a estos fueron obtenidos por Corrochano, Gómez-Gonçalves, Sevilla y Pampín-García (2017) en España, por Oh (2014) en la República de Corea o por Ucar, Cabe, y Krissek (2011) en USA.

\section{Marco metodológico}

El desarrollo de la SEA ha seguido las etapas habituales de las investigaciones de diseño. Se ha partido de una etapa preparatoria, que incluye un estudio diagnóstico y otro de diseño; hemos seguido con una etapa de implementación en dos ciclos, tras el primero de los cuales se incluyeron cambios, y finalmente se ha realizado un estudio retrospectivo a través del seguimiento de las actividades desarrolladas y la comparación del desempeño de los estudiantes antes y después de cada implementación.

Los estudios preparatorios tienen como finalidad analizar el estado de la cuestión de un determinado tema, y obtener los criterios fundamentales con los que guiar la construcción de un diseño didáctico (Molina, Castro, Molina y Castro 2011). En nuestro caso resultó complejo, pues encontramos escasos antecedentes al respecto, lo que nos obligó a realizar un estudio minucioso de diagnóstico inicial. Así, analizamos la presencia del fenómeno de las mareas en los libros de texto de secundaria, al objeto de obtener información sobre el aprendizaje previo de los estudiantes en este ámbito. Dicha información se complementó con entrevistas a profesores de ciencias de secundaria, así como de los resultados de un cuestionario previo administrado para sondear los modelos sobre el fenómeno de las mareas que empleaban estudiantes de magisterio. Finalmente, llevamos a cabo entrevistas a dos docentes de nuestra universidad que habían impartido la misma asignatura, para vislumbrar cómo incluir la nueva SEA en el contexto de su programación semestral. Toda esta información resultó clave en la elaboración de la primera versión de la SEA (SEA1), fase final del estudio preparatorio.

Una vez construida la SEA, ésta se implementó durante el curso 2018/2019 en un grupo de estudiantes $(\mathrm{n}=56)$ de tercer curso del grado en Educación Primaria de la Universidad de Cádiz, la mayoría procedentes de la provincia de Cádiz. Tras esta primera implementación, se incluyeron cambios en el diseño para obtener una segunda versión (SEA2), que fue implementada durante el curso 2019/2020 ( $n=49)$. La profesora era la misma en ambos casos y contaba con diez años de experiencia en la enseñanza de temáticas referentes al modelo S-TL, pues esa misma asignatura, en cursos anteriores, desarrolló una secuencia didáctica centrada en el fenómeno de las estaciones.

En la etapa de valoración retrospectiva recurrimos a un diseño pretest-postest con seguimiento utilizando cuestionarios escritos (Anexo I), diario de la profesora, porfolios de los alumnos, grabaciones en vídeo y audio de las sesiones y examen final cumplimentado un mes después de la finalización de la SEA. De este modo, el pretest permitió delimitar el nivel inicial de los modelos de los estudiantes sobre el fenómeno, a partir de una rúbrica elaborada a tal efecto. El postest favoreció la evaluación del nivel final alcanzado, y el examen ayudó a comprobar qué conocimientos desarrollaron después de un tiempo en el que pudieron "digerir", asentar y revisar el proceso seguido. Pero los análisis no se limitaron a un estudio 
comparativo, sino que gracias al seguimiento realizado, pudimos detectar la contribución de cada actividad al avance de los estudiantes y postular cambios para la segunda implementación.

\section{Principios de diseño para la SEA implementada}

El diseño partió de los siguientes principios:

1. Elaboración de una trayectoria de progresión desde modelos simples hacia el modelo de ciencia escolar definido.

2. Conexión entre la realidad (datos, hechos, fenómenos) y los conocimientos teóricos.

3. Secuenciación por fases del proceso de modelización.

4. Implicación activa y reflexiva del alumnado.

5. Consideración de distintos modos de organización del aula.

\section{Trayectoria de progresión}

Con el fin de prever un esquema de progresión a través de distintas etapas, consideramos útil adoptar como referente los niveles de una rúbrica elaborada en el estudio preparatorio para evaluar los modelos iniciales expresados por los estudiantes (Armario, Oliva y JiménezTenorio 2021), (ver tabla 1).

Tabla 1. Posibles niveles de progresión para el modelo de mareas

\begin{tabular}{|l|l|}
\hline Niveles & Descripción \\
\hline $\begin{array}{l}\text { 1. Modelo de causas } \\
\text { internas }\end{array}$ & $\begin{array}{l}\text { Atribuye las causas de las mareas a agentes internos a la Tierra: viento, lluvia, } \\
\text { etc.; en el contexto de un modelo topocéntrico. }\end{array}$ \\
\hline $\begin{array}{l}\text { 2.- Modelo de influencia } \\
\text { latente }\end{array}$ & $\begin{array}{l}\text { Atribuye la causa a un astro, normalmente la Luna, pero sin establecer } \\
\text { mecanismo de actuación. }\end{array}$ \\
\hline $\begin{array}{l}\text { 3.- Modelo de succión } \\
\text { gravitatoria }\end{array}$ & $\begin{array}{l}\text { Atribuye la causa a un astro, normalmente la Luna, estableciendo un } \\
\text { mecanismo explicativo basado en la fuerza gravitatoria que provocaría un } \\
\text { único abultamiento, como si, en términos metafóricos, la gravedad ejerciera un } \\
\text { efecto de arrastre o succión e implicaría la existencia de ciclos de mareas de 24 } \\
\text { h }\end{array}$ \\
\hline 4.- Modelos de transición & $\begin{array}{l}\text { Modelo de succión gravitatoria más complejo al superponer sobre él elementos } \\
\text { parciales de modelos superiores, pero sin una adecuada ni completa } \\
\text { articulación. }\end{array}$ \\
\hline $\begin{array}{l}\text { 5.- Modelo de estiramiento } \\
\text { 6odelo de dos abultamientos que llega a ser explicado metafóricamente a } \\
\text { fuerzas }\end{array}$ & $\begin{array}{l}\text { Modrir de un efecto de "estiramiento" de la masa mareal provocado por la } \\
\text { acción de la Luna. }\end{array}$ \\
\hline 7.- Modelo completo & $\begin{array}{l}\text { Modelo en el que el segundo abultamiento es explicado en términos de la } \\
\text { presencia de un gradiente de fuerza gravitatoria. }\end{array}$ \\
\hline $\begin{array}{l}\text { Modelo que se completa con la acción combinada del efecto que ejerce la Luna } \\
\text { y el Sol. }\end{array}$ \\
\hline
\end{tabular}

Así, la tabla 1 proporciona un vector de progresión que puede guiar el diseño didáctico de la propuesta de enseñanza, pero no obliga a que los estudiantes tengan que pasar por todos los modelos intermedios. De este modo, fue posible elaborar una trayectoria de progresión que contemplaba distintos hitos en el recorrido de aprendizaje:

- Hito 1. Contemplar el ciclo de mareas como un fenómeno astronómico, producido por la interacción T-L, y no como fenómeno local fruto de causas internas.

- Hito 2. Transitar de una idea de acción a distancia en la que no se especifica mecanismo de actuación, a otra similar canalizada a través de la Ley de gravitación Universal. 
- Hito 3. Trasladar la idea de un solo abultamiento a la de dos, uno a cada lado de la Tierra.

- Hito 4. Explicar la existencia de dos abultamientos a partir de un gradiente de fuerzas.

- Hito 5. Modelizar el efecto ejercido por el Sol, superpuesto al de la Luna, como origen de las mareas.

\section{Conexión entre la realidad y el conocimiento teórico}

Si bien con la edad las concepciones de los estudiantes se van adecuando a las que se enseñan en la escuela, la investigación nos dice que este tipo de conocimiento se relaciona de una forma dificultosa y fragmentaria con las experiencias cotidianas, obstaculizando la conexión entre ambas. Así, estudios sobre neurociencias revelan activaciones neuronales separadas para las percepciones implícitas y para las representaciones conceptuales explícitas (Kallai y Reiner 2010). Ello justifica la necesidad de un esfuerzo constante por familiarizar al alumnado con el fenómeno estudiado y relacionar consistentemente los hechos con los modelos generados.

\section{Secuenciación por fases del proceso de modelización}

Su diseño se estructuró por fases, en función del hito a conseguir en cada caso y de la nueva información e ideas incorporadas a través del proceso de modelización (Figura 2).

Así mismo, el sentido de estas fases se inspiró en el recorrido de los ciclos de modelización (Garrido 2016; Jorba y Sanmartí 1996; Justi y Gilbert 2002): justificación del modelo, formulación del mismo, evaluación en función de los datos, revisión e integración de nuevas ideas, aplicación, etc. Por otro lado, aunque todo el proceso se pretendía acompañar de momentos de autorregulación, pensábamos que las actividades finales deberían suponer una ocasión especial para comprobar y valorar los avances y analizar aspectos sobre la naturaleza de los modelos.

\begin{tabular}{|l||l||l|l|}
\hline DATOS, HECHOS, FENÓMENOS & FASES DE LA SEA \\
\hline Pleamar y baja mar & \begin{tabular}{|l|l|l|}
\hline Estudio de datos reales \\
mediante tablas de mareas
\end{tabular} \\
\hline astronómica
\end{tabular}

Figura 2. Fases de la secuencia didáctica y sus conexiones con hechos y modelos

\section{Implicación activa y reflexiva del alumnado}

Como ya se comentó en la introducción, los diseños didácticos basados en modelización no entienden modelizar como una actividad en la que sólo se persigue la evolución del 
conocimiento, sino donde se ofrece la oportunidad de vivenciar verdaderas prácticas científicas. Por ello, nuestro principal criterio en la secuenciación es la implicación de los estudiantes en prácticas reflexivas de modelización en ciencias. Es decir, procesos a través de los cuales el alumnado aprende y construye modelos, los revisa, reconstruye, y argumenta acerca de su valor y de su carácter parcial y limitado (Justi y Gilbert 2002). Esa reflexión es la clave sobre la que se espera una progresión en los modelos de los estudiantes, para lo que se requieren situaciones de participación activa y procesos de enseñanza donde deben aportar ideas, debatir, tomar decisiones, etc. (Oliva 2019).

\section{Distintos modos de organización del trabajo en el aula}

Otra de las premisas que preside el diseño elaborado, es la de integrar distintos modos de organización en el aula, particularmente la combinación de trabajo individual, en pequeño y gran grupo. El trabajo individual permite la reflexión inicial del estudiante ante las tareas planteadas, lo que posibilita que éste evoque un modelo o lo haga explícito. Conviene recordar que los modelos mentales de los estudiantes son implícitos e idiosincrásicos, y no siempre presentan suficiente grado de consistencia interna (Pozo, Pérez, Sanz, y Limón 1992). Por tanto, el trabajo individual ofrece ocasión para que los estudiantes verbalicen y expresen sus ideas, generando así un modelo articulado. Mientras tanto, el trabajo en pequeño grupo puede ayudar a que los estudiantes discutan y argumenten sus ideas, las hagan entrar en conflicto, y las depuren y refinen a través del consenso. Como señala Seel (2017), la conversación crea un contexto mental compartido que posibilita el intercambio de modelos personales entre los miembros de un grupo, y ello da ocasión a que se genere un modelo compartido, que suele ser más rico y coherente (Velentzas y Halkia 2013). Finalmente, el debate en gran grupo, daría ocasión a una puesta en común en la que el profesor participe aportando nuevas ideas y referentes que puedan enriquecer el discurso de los estudiantes.

\section{Resultados}

\section{Estudio preparatorio}

El estudio preparatorio ha permitido identificar muchas de las dificultades existentes en la enseñanza y aprendizaje del fenómeno de las mareas, algunas ya reflejadas en la literatura (Ballantyne 2004; Camelo, Rodríguez, y Santiesteban 2007; Corrochano et al. 2017; DelgadoSerrano y Cubilla 2012; Oh y Oh 2011; Solbes y Palomar 2011; Ucar, Trundle y Krissek 2010; Viiri 2000; Viiri y Saari 2004). Por ejemplo, pudimos constatar que la mayoría de estudiantes de magisterio se sitúan inicialmente en los modelos con niveles más bajos de la rúbrica de la tabla 1 , en concreto dentro de los tres primeros.

Por otro lado, los resultados de las entrevistas a los dos grupos de profesores nos ayudaron a entender el origen de muchas de estas dificultades: complejidad de su explicación científica; escasa conexión entre realidad y modelo manejado; presencia de modelos alternativos en los estudiantes; dificultad en el manejo de sistemas de referencia no topocéntricos y en la generación de pensamiento en $3 \mathrm{D}$, etc. Además, nos proporcionaron ideas a incorporar en el diseño objeto de estudio, dada su experiencia en la docencia en temas cercanos a éstos. Muchas de estas aportaciones reforzaron nuestro interés en el manejo de recursos como las analogías o simulaciones digitales, ya previstas en los principios de diseño antes esbozados.

El análisis de los libros de texto ayudó a conocer los antecedentes de la formación recibida por los estudiantes en su etapa como alumnos de secundaria, identificando lagunas y elementos de mejora que, tal vez, podrían ayudar a atajar algunas de las dificultades de aprendizaje señaladas. 
Es el caso, por ejemplo, de la importancia de ofrecer un enfoque integrador poniendo en valor relaciones interdisciplinares entre la física y la geografía. Esta forma de enseñanza ofrece una comprensión del fenómeno mucho más progresiva, interrelacionada y contextualizada.

Fruto de los estudios diagnósticos realizados y de los principios de diseño planteados, fue el diseño de una secuencia didáctica inicial que ha ido evolucionando y depurándose tras su primer ciclo de implementación. Al objeto de evitar reiteraciones, aportaremos solo el esquema seguido en la SEA2 (Tabla 2), ya que en la crónica de su implementación se aportarán detalles sobre las actividades incluidas y sobre las diferencias existentes entre ambas secuencias. La SEA2 completa está disponible en Armario, Jiménez-Tenorio y Oliva (2021).

Hay que indicar que las SEA se complementaron con cuatro sesiones previas destinadas a la construcción de un modelo general S-T-L, así como a contextualizar y reforzar la comprensión de la noción de modelo y de tres fenómenos astronómicos muy relacionados con el fenómeno de las mareas: el día/noche, las fases de la Luna y los eclipses.

Tabla 2. Secuencia didáctica empleada en la experiencia

\begin{tabular}{|c|c|c|c|c|c|}
\hline Sesión & Fase & Actividad & Descripción & Propósito didáctico & Recursos \\
\hline 1 & \multirow[b]{2}{*}{$\begin{array}{c}\text { Explorando las } \\
\text { ideas }\end{array}$} & $\begin{array}{l}\text { 1. Cuestionario } \\
\text { inicial }\end{array}$ & $\begin{array}{l}\text { Caracterización de los } \\
\text { modelos de partida }\end{array}$ & $\begin{array}{l}\text { Explicitar los modelos } \\
\text { empelados por los } \\
\text { alumnos }\end{array}$ & Cuestionario \\
\hline 2 & & $\begin{array}{l}\text { 2. ¿Conocemos } \\
\text { las mareas? }\end{array}$ & $\begin{array}{l}\text { Comparación de sus } \\
\text { dibujos y/o } \\
\text { explicaciones y } \\
\text { clasificación de estos }\end{array}$ & $\begin{array}{l}\text { Contraste con nuevas } \\
\text { visiones. Tomar } \\
\text { conciencia del uso de } \\
\text { modelos }\end{array}$ & $\begin{array}{l}\text { Dibujos } \\
\text { realizados por } \\
\text { ellos mismos }\end{array}$ \\
\hline \multirow[b]{2}{*}{3} & \multirow[b]{2}{*}{$\begin{array}{l}\text { Hacia una visión } \\
\text { astronómica }\end{array}$} & $\begin{array}{l}\text { 3. ¿Cada cuánto } \\
\text { tiempo? }\end{array}$ & $\begin{array}{l}\text { Representación de } \\
\text { datos reales sobre } \\
\text { pleamar y bajamar }\end{array}$ & $\begin{array}{l}\text { Tomar consciencia de la } \\
\text { periodicidad del } \\
\text { fenómeno }\end{array}$ & $\begin{array}{l}\text { Manejo de } \\
\text { tablas de } \\
\text { mareas }\end{array}$ \\
\hline & & $\begin{array}{l}\text { 4. Hagamos } \\
\text { nuestro modelo }\end{array}$ & $\begin{array}{l}\text { Representación de los } \\
\text { modelos causales de las } \\
\text { mareas }\end{array}$ & $\begin{array}{l}\text { Explicitación del efecto } \\
\text { lunar. Contraste entre las } \\
\text { conclusiones anteriores y } \\
\text { los modelos } \\
\text { representados }\end{array}$ & Papel y lápiz \\
\hline \multirow[b]{2}{*}{4} & \multirow{5}{*}{$\begin{array}{l}\text { Representando y } \\
\text { reestructurando }\end{array}$} & 5. Muelle e imán & Analogía & $\begin{array}{l}\text { Visualizar los dos } \\
\text { abultamientos de marea }\end{array}$ & \multirow{2}{*}{$\begin{array}{l}\text { Imán de } \\
\text { neodimio y } \\
\text { muelle }\end{array}$} \\
\hline & & & & $\begin{array}{l}\text { Crear conflicto con sus } \\
\text { ideas intuitivas }\end{array}$ & \\
\hline 5 & & $\begin{array}{l}\text { 6. Tren de } \\
\text { carritos }\end{array}$ & Analogía & $\begin{array}{l}\text { Comprender la causa de } \\
\text { las mareas (dos } \\
\text { abultamientos por } \\
\text { estiramiento) }\end{array}$ & $\begin{array}{l}\text { Sistema de } \\
\text { carritos }\end{array}$ \\
\hline \multirow[b]{2}{*}{6} & & $\begin{array}{l}\text { 7. ¡Ponte en } \\
\text { situación! }\end{array}$ & Analogía & $\begin{array}{l}\text { Comprender la causa de } \\
\text { las mareas (dos } \\
\text { abultamientos por } \\
\text { estiramiento) }\end{array}$ & $\begin{array}{l}\text { Personificació } \\
\mathrm{n} \text { de los dos } \\
\text { abultamientos } \\
\text { mareales }\end{array}$ \\
\hline & & $\begin{array}{l}\text { 8. Diagrama de } \\
\text { correspondencia }\end{array}$ & $\begin{array}{l}\text { Comparación de las } \\
\text { tres analogías }\end{array}$ & $\begin{array}{l}\text { Síntesis sobre la causa de } \\
\text { los dos abultamientos } \\
\text { mareales }\end{array}$ & $\begin{array}{l}\text { Tabla } \\
\text { comparativa }\end{array}$ \\
\hline \multirow{4}{*}{7} & \multirow{4}{*}{ Ampliando visión } & 9. ¿Qué pasaría? & Experimento mental & $\begin{array}{l}\text { Introducir el Sol en el } \\
\text { fenómeno }\end{array}$ & $\begin{array}{l}\text { Discusión en } \\
\text { grupo }\end{array}$ \\
\hline & & $\begin{array}{l}\text { 10. Mareas vivas y } \\
\text { muertas }\end{array}$ & $\begin{array}{l}\text { Lecturas y reflexión de } \\
\text { noticias de prensa }\end{array}$ & $\begin{array}{l}\text { Conceptualizar las } \\
\text { mareas vivas y muertas }\end{array}$ & $\begin{array}{l}\text { Extractos de } \\
\text { noticias }\end{array}$ \\
\hline & & 11. Simulación & $\begin{array}{l}\text { Simulación en webs de } \\
\text { mareas vivas y muertas }\end{array}$ & $\begin{array}{l}\text { Comprobar las } \\
\text { conclusiones del } \\
\text { experimento mental }\end{array}$ & \multirow[t]{2}{*}{$\begin{array}{l}\text { Animación } \\
\text { digital }\end{array}$} \\
\hline & & & & $\begin{array}{l}\text { Analizar diferencias entre } \\
\text { modelo inicial y actual }\end{array}$ & \\
\hline
\end{tabular}


Tabla 2. Continuación

\begin{tabular}{|c|c|c|c|c|c|}
\hline Sesión & Fase & Actividad & Descripción & Propósito didáctico & Recursos \\
\hline 8 & \multirow{4}{*}{$\begin{array}{l}\text { Llegando a la } \\
\text { meta }\end{array}$} & $\begin{array}{l}\text { 12. Sol, Tierra, } \\
\text { mar ja dibujar! }\end{array}$ & $\begin{array}{l}\text { Realizar un dibujo } \\
\text { explicativo sobre el } \\
\text { fenómeno de las } \\
\text { mareas }\end{array}$ & $\begin{array}{l}\text { Reconstrucción del } \\
\text { modelo }\end{array}$ & Papel y lápiz \\
\hline \multirow{2}{*}{9} & & $\begin{array}{l}\text { 13. ¡Hora de } \\
\text { aplicar el } \\
\text { conocimiento! }\end{array}$ & $\begin{array}{l}\text { Resolución de un } \\
\text { problema }\end{array}$ & $\begin{array}{l}\text { Aplicar el modelo para } \\
\text { hacer predicciones }\end{array}$ & $\begin{array}{l}\text { Problema de } \\
\text { predicción }\end{array}$ \\
\hline & & mareas? & $\begin{array}{l}\text { Observar el relieve } \\
\text { costero del océano } \\
\text { atlántico y el mar } \\
\text { mediterráneo }\end{array}$ & $\begin{array}{l}\text { Reflexionar sobre agentes } \\
\text { potenciadores de las } \\
\text { mareas }\end{array}$ & Mapamundi \\
\hline 10 & & 16. Postest & $\begin{array}{l}\text { Caracterización de los } \\
\text { modelos finales }\end{array}$ & $\begin{array}{l}\text { Explicitar los modelos de } \\
\text { los estudiantes tras la } \\
\text { implementación de la } \\
\text { secuencia }\end{array}$ & Cuestionario \\
\hline
\end{tabular}

\section{Crónica de la implementación}

La crónica de implementación se describirá en cinco fases indicadas en la tabla 2, cuyos títulos dan sentido al contenido de cada una y servirán para situar mejor la reconstrucción del camino recorrido. En la primera, explorando las ideas, se analizaron los modelos iniciales de los alumnos. En la segunda, hacia una visión astronómica, evaluamos el tránsito esperado desde una visión interna del fenómeno a otra astronómica. Seguidamente, en representado y reconstruyendo, analizamos el papel de recursos analógicos en la evolución hacia modelos más complejos. La cuarta, ampliado visión, permitió situar el papel del Sol tomando como referencia otros eventos como las mareas vivas y muertas y, por último, llegando a la meta, favoreció el balance de los logros alcanzados y de las metas por alcanzar, a través de la reconstrucción de la puesta en común final de la SEA.

\section{Explorando las ideas}

En las dos primeras sesiones, los alumnos desarrollaron actividades exploratorias al fin de visibilizar sus modelos iniciales sobre este tópico, así como hacerles conscientes de las lagunas que pudieran tener al respecto. Para ello se recurrió al mismo cuestionario utilizado en el estudio preparatorio. En la segunda implementación se decidió incorporar una puesta en común tras su desarrollo, para facilitar el contraste de ideas entre compañeros y favorecer su expresión oral, la cual resultó de mayor claridad que la escrita.

El debate generado, junto con la información del cuestionario escrito, reveló dificultades a la hora de describir el fenómeno, sobre todo para establecer su periodicidad, ya que muchos estudiantes apuntaban a ciclos de mareas de $24 \mathrm{~h}$. En este sentido, las ideas surgidas fueron muy variadas en contenido y complejidad, ajustándose casi todas a los tres primeros niveles de la rúbrica de la tabla 1 . Unos alumnos señalaban causas internas, como el viento o las corrientes marinas. En este caso, normalmente se reflejaba una visión local o topocéntrica, interna, parcial y muy alejada del modelo de ciencia escolar. Otros asociaban el fenómeno con la Luna sin establecer relación causal alguna, mientras los restantes llegaban a considerar a la fuerza gravitatoria lunar como el principal agente causante; si bien lo concebían en términos de acumulación de agua en la franja de la Tierra más próxima a la Luna. No todos los 
estudiantes integrados en este grupo señalaban como agente causante a esta fuerza, existiendo algunos que mencionaban únicamente a la gravedad como responsable, sin establecer relación con el cuerpo causante de la misma. Por último, solo una mínima parte conceptualizaron el fenómeno en términos de un modelo de transición. Éstos lo hicieron a través de un modelo semejante al de succión gravitatoria, pero al que se añadía la existencia de dos abultamientos, en vez de uno, pero sin aportar una explicación convincente de ello. Todas estas ideas ya fueron encontradas en el estudio preparatorio.

La puesta en común de sus respuestas al cuestionario incial hizo visible el escaso dominio de los conocimientos descriptivos asociados al fenómeno, a pesar de proceder el alumnado, en su mayoría, de poblaciones costeras. Esto sugería otra dificultad en su modelización, ya que indicaba un desconocimiento de la base empírica de referencia necesaria para construir un modelo teórico. Por otra parte, la incoherencia entre las explicaciones causales, donde se reflejaba una visión astronómica con la Luna como protagonista, y los dibujos realizados en la pizarra donde hacía alusión a la playa, indicaba la dificultad para cambiar el sistema de referencia a otro más abstracto y alejado de su experiencia diaria.

Con respecto a la segunda actividad, ¿Conocemos las mareas?, donde debían agrupar y discutir sobre los dibujos realizados en la actividad anterior, se observaron limitaciones para clasificar, ilustrar y ordenar ideas tipo sobre el fenómeno estudiado, pues la mayoría no realizó ningún dibujo en el cuestionario inicial. Aquellos estudiantes que sí lo intentaron, hicieron representaciones muy simples, y mayoritariamente representativas de la línea de costa. Esto, sin duda, supuso un obstáculo para el adecuado desarrollo de la actividad ya que debían hacer uso de dichos dibujos.

\section{Hacia una visión astronómica}

La tercera sesión tenía dos objetivos diferenciados. De un lado, se pretendía trabajar aspectos fenomenológicos del proceso natural. Del otro, se procuró favorecer una visión astronómica focalizada en la influencia de la Luna (Hito 1).

Para el primero de ellos, los estudiantes trabajaron con tablas de mareas simplificadas, lo que nos permitió detectar problemas relacionados con el tratamiento de la información: ejes cartesianos, variables dependientes e independientes, construcción de escalas, etc. En ella indicábamos la variación del nivel del mar $(\mathrm{m})$ en función del tiempo (h) en una localidad concreta. En total recogíamos la variación dada en $48 \mathrm{~h}$. La actividad permitió comprobar visualmente unas de las conclusiones más relevantes hasta el momento: la existencia de dos pleamares y dos bajamares al cabo de $24 \mathrm{~h}$. Esta idea es esencial para la construcción de un modelo con dos abultamientos mareales (Figura 1). Además, esta sesión favoreció la contextualización del fenómeno en el día a día del alumnado, ya que la actividad facilitó que recordasen experiencias vividas en torno al mismo.

La segunda parte de la sesión se dedicó a favorecer una visión causal del fenómeno ofreciendo un mecanismo explicativo basado en la interacción gravitatoria. Así, a través de la actividad Hagamos nuestro modelo, presentamos un pequeño relato de la evolución del conocimiento sobre el fenómeno a lo largo de la historia. En él resaltábamos los principales hitos sobre la causalidad de las mareas (Hito 2), como por ejemplo las conclusiones científicas del explorador Phytheas (330 a. C) sobre la relación entre las mareas y la Luna o las ofrecidas por Newton, responsable de dar solución científica al fenómeno. La figura 3 muestra un dibujo de la interpretación que hizo uno de los estudiantes. Puede verse que, aunque ya el fenómeno es explicado en términos de atracción de la Luna, todavía la representación se sitúa en un modelo de succión gravitatoria, que concibe un solo abultamiento e implica ciclos de mareas de $24 \mathrm{~h}$. 


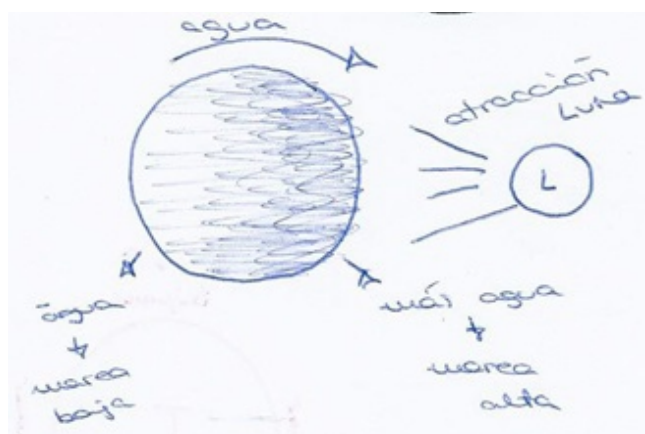

Figura 3. Modelo de un solo abultamiento mareal propuesto por el grupo 6

La explicitación mediante dibujos de un modelo de succión con un solo abultamiento, supuso una ocasión para generar una situación de conflicto cognitivo entre sus predicciones y la realidad de los hechos de ciclos de $12 \mathrm{~h}$. Consecuencia de ello fue que algunos estudiantes empezaron a sopesar la plausibilidad de un modelo con dos abultamientos, uno a cada lado de la Tierra (Hito 3). Sin embargo, ninguno fue capaz de explicar con precisión la causalidad de dicho fenómeno en términos de la existencia de un gradiente de fuerzas.

\section{Representando y reestructurando}

Las sesiones 4, 5 y 6 abordaron el hito 4, el cual preveíamos de mayor dificultad al considerar el origen del segundo abultamiento mareal. Para ello se utilizó una analogía múltiple en la que, a partir de tres símiles, se pretendía modelizar el elipsoide de revolución formado por la masa de agua, en términos de una esfera estirada por los extremos por el efecto de un diferencial de fuerza entre los dos puntos opuestos de su diámetro horizontal. Estas analogías supusieron en conjunto una herramienta útil para realizar un acercamiento al modelo de ciencia escolar, a través de la comparación de análogos más cercanos al alumnado como imanes, muelles y carritos de juguete. En la tabla 3 se aporta un esquema del modelo trabajado sobre el fenómeno de las mareas y los correspondientes modelos analógicos, así como sus intenciones didácticas, semejanzas y diferencias. En todos los casos, se pretendía hacer patente la deformación por estiramiento ocasionada en un sistema flexible al ser sometido a un diferencial de fuerza entre dos puntos extremos.

En la primera actividad analógica, los estudiantes debían manipular un imán y un muelle con el fin de recrear el sistema T-L y las fuerzas de atracción ejercidas entre ambos. Concretamente, se trataba de establecer la existencia de un gradiente de fuerzas como causa de la deformación de la masa oceánica, a partir del establecimiento de relaciones analógicas y visualizar el efecto de éste sobre la masa oceánica. En este caso, este efecto se traduce en el estiramiento del muelle, debido a la disminución de la fuerza magnética con la distancia producida por el imán. Para ello podían hacer uso de una pinza con el fin de retener el muelle por un extremo y facilitar la visualización de la diferencia de estiramiento.

Posteriormente la actividad contemplaba un espacio para el anáisis de las limitaciones de la comparación. Observamos que la analogía sirvió más para crear debate sobre aspectos propios de la modelización que para cumplir su objetivo. De hecho, se obtuvieron dificultades a dos niveles: a) en la comprensión del sentido de la analogía, y b) en la transferencia analógica del análogo al objeto. 
Tabla 3. Modelo trabajado, analogías utilizadas, semejanzas y diferencias

\begin{tabular}{|c|c|c|c|}
\hline \multirow{3}{*}{$\begin{array}{l}\text { Fenómeno a modelar: } \\
\text { segundo abultamiento mareal }\end{array}$} & \multicolumn{3}{|c|}{ Modelos análogos } \\
\hline & $\begin{array}{c}\text { Sistema de imán y } \\
\text { muelle }\end{array}$ & $\begin{array}{l}\text { Sistema de carritos } \\
\text { unidos por una cuerda }\end{array}$ & Personificación \\
\hline & 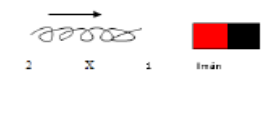 & \%क के & $\overbrace{\text { Puato2 }}^{2} \overbrace{\text { Putas }}^{2}$ \\
\hline Descripción del sistema & $\begin{array}{l}\text { Un imán deforma } \\
\text { un muelle. El imán } \\
\text { atrae } \\
\text { particularmente a la } \\
\text { parte más cercana, } \\
\text { lo que provoca que } \\
\text { ésta se acerque y el } \\
\text { muelle se deforme. }\end{array}$ & $\begin{array}{l}\text { Un sistema de carritos } \\
\text { unidos por cuerdas } \\
\text { destensadas se estira } \\
\text { cuando una mano tira } \\
\text { del carrito de un } \\
\text { extremo. }\end{array}$ & $\begin{array}{l}\text { E1/la alumno/a que } \\
\text { escenifica la Luna, pide a } \\
\text { los otros alumnos que se } \\
\text { acerquen a él/ella, en el } \\
\text { caso del punto } 1 \text { dando } \\
\text { tres pasos, en el del punto } \\
\mathrm{X} \text { dando dos, y en el del } \\
\text { punto } 2 \text { dando un paso: } \\
\text { Es decir, avanza más el } \\
\text { más cercano y menos los } \\
\text { más distantes. }\end{array}$ \\
\hline Intención didáctica & \multicolumn{3}{|c|}{$\begin{array}{l}\text { Los tres análogos intentan representar el efecto de estiramiento que produce } \\
\text { la acción gravitatoria de la Luna sobre la masa oceánica, debido al gradiente } \\
\text { de fuerzas que actúa sobre distintos puntos (señalados con } 1 \text { y 2) de ésta } \\
\text { última. Esto facilita la conceptualización de los abultamientos mareales y, } \\
\text { sobre todo, el segundo (el que se encuentra más alejado de la Luna). }\end{array}$} \\
\hline Correspondencias & $\begin{array}{l}\text { - E1 imán } \\
\text { representa a la } \\
\text { Luna. } \\
\text { - E1 muelle } \\
\text { correspond } \\
\text { e a la masa } \\
\text { oceánica. } \\
\text { - E1 punto X } \\
\text { visualiza el centro } \\
\text { de la Tierra. }\end{array}$ & $\begin{array}{l}\text { - La mano que tira de } \\
\text { un extremo } \\
\text { corresponde con la } \\
\text { Luna. } \\
\text { - El sistema de carros } \\
\text { unidos por cuerdas se } \\
\text { considera similar a la } \\
\text { masa oceánica, siendo } \\
\text { el central el que } \\
\text { equivaldría a la Tierra. }\end{array}$ & $\begin{array}{l}\text { - Un/a de los/as } \\
\text { alumnos/as hace el papel } \\
\text { de Luna. } \\
\text { - Los/as restantes unidos } \\
\text { por cuerdas escenificarían } \\
\text { la Tierra y la masa } \\
\text { oceánica que la envuelve. }\end{array}$ \\
\hline Similitudes & \multicolumn{3}{|c|}{$\begin{array}{l}\text { Objeto y análogos están formados por dos elementos: un agente que provoca } \\
\text { una acción que varía con la distancia y un sistema volumétrico flexible que se } \\
\text { estira, debido al gradiente de fuerzas que lo deforma. }\end{array}$} \\
\hline \multirow[t]{2}{*}{ Diferencias } & \multicolumn{3}{|c|}{$\begin{array}{l}\text { - Sistemas visualmente diferentes. Los sistemas analógicos no manifiestan } \\
\text { una deformación espacial sino longitudinal. } \\
\text { - El gradiente de fuerzas solo es representado de forma cualitativa. } \\
\text { - Sistemas incompletos. Ninguna de las analogías contempla el Sol como } \\
\text { otro elemento del fenómeno de las mareas. }\end{array}$} \\
\hline & $\begin{array}{l}\text { - La interacción es } \\
\text { magnética, no } \\
\text { gravitatoria. } \\
\text { - El imán y el } \\
\text { muelle pueden } \\
\text { llegar a colisionar. }\end{array}$ & $\begin{array}{l}\text { - La acción la ejerce la } \\
\text { mano, no una fuerza a } \\
\text { distancia. } \\
\text { - Solo actúa fuerza } \\
\text { sobre el carro más } \\
\text { cercano a la mano, al } \\
\text { menos hasta que las } \\
\text { cuerdas se tensan. }\end{array}$ & $\begin{array}{l}\text { - En la personificación, la } \\
\text { acción no la desencadena } \\
\text { una fuerza, sino las } \\
\text { instrucciones que da el/la } \\
\text { alumno/a que escenifica } \\
\text { la Luna. } \\
\text { - Las instrucciones } \\
\text { pueden parecer arbitraria. }\end{array}$ \\
\hline
\end{tabular}

A lo largo de la intervención se obtuvieron numerosos registros que ilustraban las dificultades encontradas por los estudiantes. Un ejemplo de ello lo encontramos en la conclusión formulada por uno de los grupos en la que apreciamos una argumentación coherente con el modelo de succión gravitatoria lunar:

"El imán funcionaría como si fuera la Luna, y el muelle la Tierra. La fuerza de atracción que ejerce el imán sobre el muelle es igual que la atracción de la Luna sobre la Tierra. 
Esta, gracias a la fuerza gravitatoria, atrae el nivel del agua de los mares y océanos, por lo que dicho nivel aumenta o disminuye según la cercanía de la Luna con la Tierra".

Para la segunda analogía (tabla 3), utilizamos como análogo un sistema de tres carritos de juguetes, unidos entre sí con cuerdas destensadas. Su objetivo también era hacer visible el efecto de gradiente de fuerzas entre distintos puntos de un sistema. $\mathrm{Y}$ es que al tirar del extremo (cuerda sobrante) del carrito 1, los tres se van desplazando sucesivamente hasta que las cuerdas se tensan. En este caso, la visualización del gradiente fue más accesible, ya que los análogos eran más fáciles de manipular y el alumnado tenía el antecedente de la analogía anterior, lo cual favoreció que se fijara con detalle en las diferencias de desplazamientos entre los carritos. Además, en esta ocasión debían hacer marcas iniciales y finales de estos desplazamientos, favoreciendo así su tarea. Aun así, la mayoría de los estudiantes no consiguieron alcanzar el objetivo deseado.

Dado que las dificultades de comprensión persistían, se decidió incorporar una tercera analogía en forma de personificación (López 2014). Ésta consistía en adjudicarle a distintos estudiantes un rol dentro del sistema T-L. Concretamente, en ella intervinieron cuatro alumnos: tres formaron el sistema Tierra (los dos abultamientos mareales y el núcleo del planeta) y el restante adoptó la función de la Luna. El efecto de ésta última se representó mediante desplazamientos relativos de los estudiantes. Dichos desplazamientos estaban en función de la fuerza que supuestamente ejercería la persona que realiza el papel de la Luna, en función de su distancia: el más cercano se desplazaba tres pasos hacia ella por ser el que recibía una fuerza mayor (n.1), el que adoptó el rol de núcleo de la Tierra dos pasos por asumir una fuerza más pequeña $\left(\mathrm{n}^{\circ} .2\right)$ y el último, sólo uno por ser el que recibiría menor fuerza $\left(\mathrm{n}^{\circ} .3\right)$ (Figura 4). De este modo, a través de la separación entre los estudiantes se visualizaba el efecto de estiramiento que origina el diferencial de fuerza gravitatoria provocado por la Luna sobre el sistema Tierra.

El hecho de que los estudiantes se convirtieran en protagonistas activos de la analogía, parece que ayudó a que entendieran su mensaje y que contribuyera más a la transferencia analógica que los símiles anteriores.
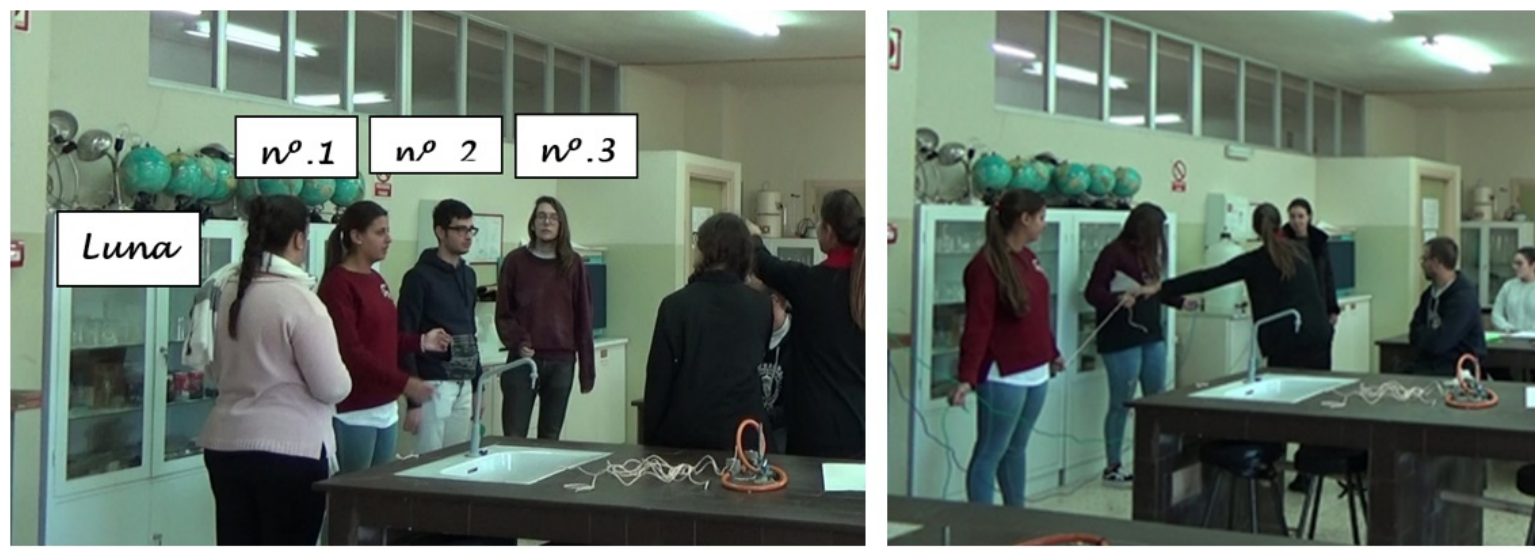

Figura 4. Distribución de roles en la personificación

Para cerrar la microsecuencia analógica, en la segunda implementación se llevó a cabo una síntesis, a través de una tabla comparativa, entre los elementos de las tres analogías y el fenómeno de las mareas. El objetivo era ayudar a la reconstrucción de los modelos de los alumnos, mediante la reflexión de las conclusiones obtenidas a través de estas analogías. Se observaron las mismas dificultades anteriores, si bien en esta ocasión la labor de andamiaje 
realizada por la profesora consiguió que parte de los estudiantes sí sacaran partido de la actividad realizada (Figura 5).

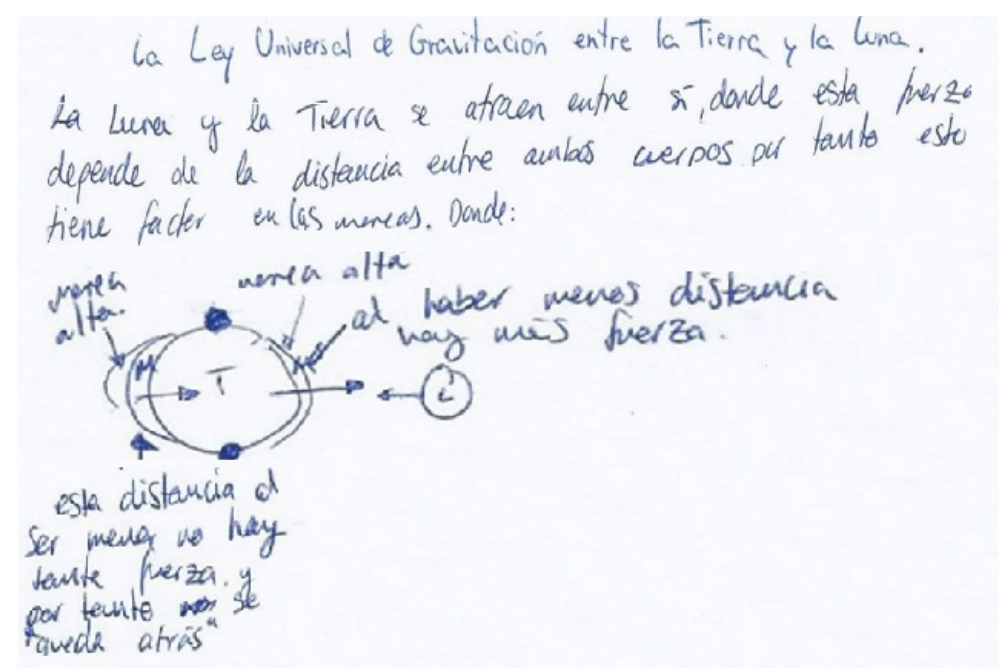

Figura 5. Portafolios de uno de los grupos tras finalizar las actividades analógicas

En conjunto, obtuvimos mejores resultados con la personificación que con las otras dos analogías. Sin embargo, también hay que decir que la personificación fue la última comparación en implementarse, por lo que los alumnos contaban ya con el precedente de las anteriores. Por otra parte, y dejando a un lado los resultados de cada analogía, todas ellas fomentaron la participación y discusión de los estudiantes, quienes se implicaron notablemente.

\section{Ampliando visión}

Las siguientes actividades se centraron en el objetivo de estudiar la combinación de efectos ejercidos por el Sol y la Luna (Hito 5). Para conseguirlo, la sesión siete comenzó con un experimento mental donde los alumnos debían incluir un nuevo elemento dentro de su modelo y evaluar sus efectos. Para implicar a los estudiantes se plantearon las preguntas: ¿Qué pasaria si en el sistema Tierra-Luna interviniera también el Sol? ¿Qué efecto provocaría en el fenómeno de las mareas? Explicadlo. Ello situó al alumnado ante el reto de realizar un experimento mental, un tipo de actividad sumamente interesante, sobre todo cuando se realiza en el contexto grupal (Reiner y Gilbert, 2000).

Un aspecto esencial en este momento fue la alusión a los fenómenos de mareas vivas y muertas, para lo que se aportaron noticias de prensa. Estos documentos pretendían ayudar a que los estudiantes imaginasen la superposición del efecto de los dos astros, dando así cabida en sus explicaciones a estos hechos. Los alumnos tuvieron dificultades para realizar la tarea porque confundían mareas vivas y muertas con pleamar y bajamar, como se demuestra en este extracto del porfolio de unas alumnas:

"La [marea] viva se da cuando hay mucha fuerza, entonces crece más y la marea es mayor y la [marea] muerta es cuando está en bajamar, tiene muy poca fuerza entonces es como si no se moviera" (Ana).

Por esta razón, el desarrollo del experimento mental se complementó con una simulación por ordenador, extraída de la página web del National Oceanic and Atmospheric Administration (NOAA) (https://oceanservice.noaa.gov/education/kits/tides/media/supp_tide06a.html).

Aunque la simulación sirvió para sus propósitos, al menos de manera parcial, la dificultad del fenómeno invitaba a la realización de una nueva personificación en el aula para la que se pidió 
voluntarios, algo que surgió como una actividad espontánea. Tres estudiantes se prestaron a ello: uno representó la Tierra, otro la Luna y otro más el Sol. Mientras la profesora iba preguntando sobre la disposición de los elementos del sistema S-T-L durante las mareas vivas y muertas, los alumnos se iban disponiendo libremente según sus correspondientes roles. Esta actividad, favoreció la puesta en marcha y aplicación de los modelos de muchos estudiantes. De una forma dinámica y participativa, los alumnos revisaron, autorregularon y reconstruyeron sus modelos, generando un ambiente motivador y una sensación de aprendizaje generalizada. A raíz de estas discusiones surgió una de las dudas más comunes, la relación entre las fases lunares, los eclipses y los fenómenos de mareas vivas y muertas.

A partir de aquí, las conversaciones generadas se volvieron más participativas y enriquecedoras, llegando incluso a constituirse espontáneamente pequeños grupos de debate. $\mathrm{Si}$ bien no todos mostraron total comprensión del fenómeno, esta actividad ayudó notablemente a avanzar en la dirección deseada.

\section{Llegando a la meta}

Se desarrollaron actividades de consolidación, aplicación y metacognición en torno al modelo construido. Así, a través de las tres últimas sesiones los alumnos realizaron, por un lado, dibujos y pequeñas maquetas de plastilina al objeto de concretar el modelo desarrollado (Viiri y Saari 2004) (Figura 6). Por otro, aplicaron sus modelos mediante la resolución de problemas en contextos reales, autorregulando el conocimiento generado en función de las respuestas ofrecidas. Por ejemplo, hicieron predicciones sobre el estado de la marea en una playa en función del tiempo transcurrido a partir de una situación concreta, diferenciaron el fenómeno de las mareas y otros procesos naturales, como las tormentas, y explicaron el diferente comportamiento de las mareas en el Atlántico y Mediterráneo.

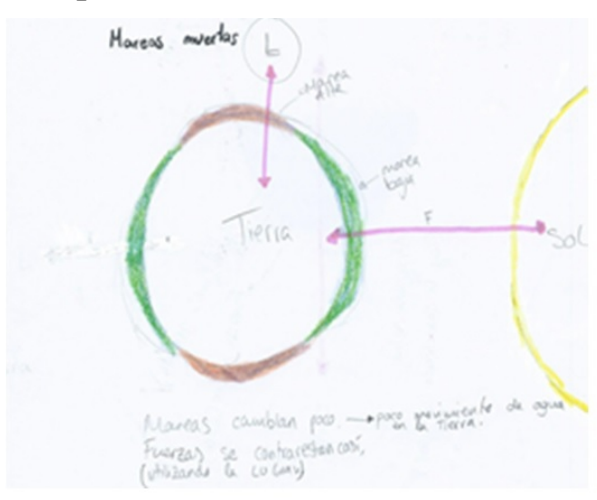

(a)

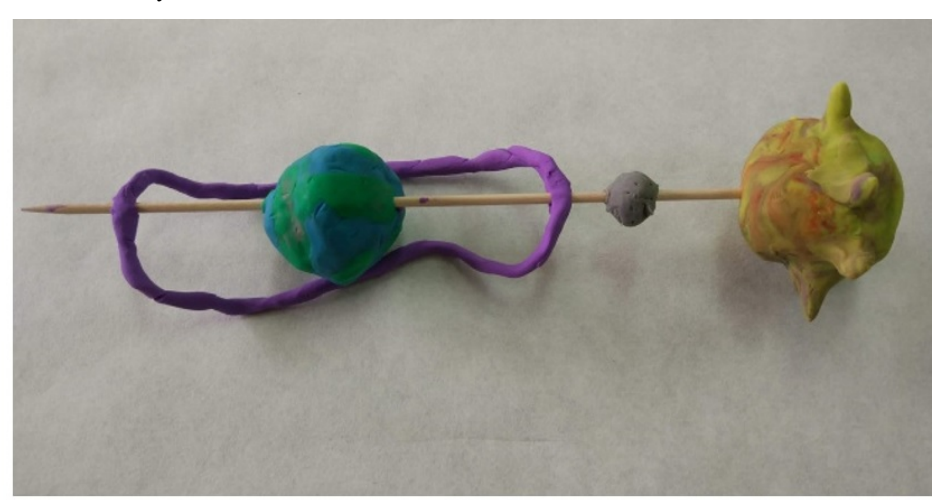

(b)

Figura 6. Dibujos y modelos 3D con plastilina para representar el efecto conjunto de la Luna y el Sol

En la penúltima sesión, los estudiantes, a través de la puesta en común de los dibujos realizados en la sesión anterior, sintentizaron lo aprendido. Otra de sus intenciones era conseguir la autorregulación y evaluación de los modelos individuales de cada alumno, a través de la evaluación de los modelos de sus compañeros. Así, había estudiantes que todavía reflejaban ciertas dudas sobre la comprensión del concepto de gradiente de fuerza, y otros que cada vez se sentían más cómodos discutiendo sobre el tema. Incluso algunas alumnas fueron capaces de comparar características de varios modelos a la vez y analizar sus detalles.

Además, y aunque durante toda la SEA se insistió en la naturaleza de los modelos, mediante esta síntesis final se volvió a hacer hincapié en su carácter parcial y limitado. Sin embargo, no se obtuvieron muchas reflexiones al respecto. Quizás esto tenga que ver con la orientación de las actividades propuestas o la formulación de sus enunciados, pues en las primeras actividades de la secuencia sí se obtuvieron reflexiones explícitas relacionadas. Sin embargo, a medida que 
iba desarrollándose el proceso didáctico, este aspecto iba ocupando un segundo plano, adquiriendo más importancia las discusiones sobre el contenido científico. No obstante, y propiciado por la profesora, se consiguió discutir sobre el paralelismo entre la evolución del conocimiento a lo largo de la historia y su propia evolución sobre el fenómeno de las mareas, del tal forma que algunos alumnos fueron capaces de reconocer una evolución en sus ideas.

Finalmente, en la última sesión los alumnos respondieron al mismo cuestionario sobre las mareas utilizado en la primera sesión como instrumento de exploración de ideas previas. Un mes más tarde tuvo lugar el examen que sirvió como nueva ocasión para evaluar los modelos desarrollados por los estudiantes.

\section{Análisis retrospectivo}

Los instrumentos de seguimiento, como el diario de la profesora y el porfolio, sirvieron para pulsar el proceso de implementación y hacer cambios sobre la marcha. Muchos de los resultados han sido presentados a lo largo de la crónica, y sugieren que la SEA contribuyó a la progresión de los modelos de los estudiantes, no sin dificultades y sin que podamos decir que dicha progresión afectara por igual a la totalidad del alumnado.

Además, esos instrumentos sirvieron para decidir cambios en la SEA1 hasta desembocar en la SEA2. Así, se generaron dos tipos de modificaciones tras el primer ciclo de implementación. Unas relacionadas con la redacción y el estilo de las preguntas y otras más centradas en el tipo de actividades y su correspondiente secuenciación. Con respecto a la primera, en la SEA1 se observó cierta dificultad para comprender algunos de los enunciados, por lo que decidimos sustituir los términos problemáticos por otros sinónimos más cercanos al alumnado. Por ejemplo, el término "gradiente de fuerzas" se sustituyó por el de "diferencial de fuerzas". Además, nos percatamos de que habíamos desgranado demasiado las actividades a través de preguntas cortas y separadas, lo que ofrecía una sensación fragmentaria de las tareas. Por ello, en el segundo diseño optamos por unirlas y presentarlas todas en un mismo enunciado.

En cuanto a los cambios relacionados con las actividades y su secuenciación, se modificó el orden de actuación de alguna de las actividades, se varió la presentación de los datos empleados en algunas de las sesiones y, por último, se eliminaron algunas actividades de la propuesta y se añadieron otras con el objetivo de fortalecer los puntos débiles detectados en la SEA1.

Finalmente, tras acabar los dos ciclos de implementación se realizó un estudio más a fondo, en el que se compararon los resultados de los dos cuestionarios (previo y posterior a la SEA) y el examen final. Ello permitió realizar un balance global de logros conseguidos. Si bien en otro artículo que tenemos en preparación se llevará a cabo un análisis exhaustivo para dichas comparaciones, resulta obligado en éste formular un breve avance. Los resultados muestran progresión en los modelos manejados por los participantes. Casi dos tercios del alumnado mantuvo inicialmente modelos compatibles con los niveles 2 y 3 de la tabla 1 , siendo muy pocos los que alcanzaron el nivel 4, y ninguno modelos más avanzados. Por el contrario, en el cuestionario final, alrededor del 70\% se situaba en el nivel 4, y el resto se repartió entre los niveles superiores, alcanzando uno de cada 20 alumnos aproximadamente el nivel 7, el más alto de la rúbrica. Pero, a pesar de la mejoría en los modelos expresados, se apreció una progresión extra al cabo de un mes después, en el examen de la asignatura. Casi dos tercios del alumnado fue capaz de alcanzar los niveles 5, 6 y 7, situándose, casi un tercio, en el nivel superior. Por tanto, el proceso de aprendizaje continuó después de acabar la SEA. 
En resumen, creemos disponer de suficientes evidencias que sugieren la utilidad de la SEA implementada en la progresión de los modelos de los estudiantes sobre el fenómeno estudiado.

\section{Discusión}

Como ya indicamos, la secuencia didáctica descrita y evaluada constituye en sí misma un producto de la investigación desarrollada. Si bien su uso no es generalizable para cualquier contexto, sí es extensible a otros casos de formación inicial del profesorado, particularmente de lugares costeros. Incluso contiene aspectos que podrían extrapolarse a la educación secundaria, ya que muchos de los elementos que la integran estaban pensados para un abordaje en la educación secundaria obligatoria (Armario, Oliva, y Jiménez-Tenorio 2019).

Por otro lado, los resultados obtenidos contribuyen a validar los principios que sustentaron el diseño de partida. La trayectoria de progresión planificada resultó adecuada para los modelos iniciales de los participantes y supuso un marco útil para la secuenciación de actividades (Acher 2014; Clement 2000). Además, la incorporación de fases de aprendizaje basadas en el marco de modelización (Halloun 2007; Justi y Gilbert 2002) resultó conveniente para establecer un hilo conductor, y sirvió como ocasión para implicar activa y reflexivamente al alumnado en prácticas de modelización en ciencias.

Asimismo, los datos obtenidos muestran la importancia de establecer conexiones entre la realidad y el modelo elaborado para representarla. Por ejemplo, las tablas de mareas resultaron determinantes para consolidar la idea de ciclos de mareas de $12 \mathrm{~h}$, mientras que las analogías, personificaciones, simulaciones y experimentos mentales constituyeron recursos que posibilitaron la conexión entre dichos ciclos y un modelo teórico. Esta conexión resultó esencial para poner a prueba, revisar y mejorar los modelos manejados. Se trataba de evitar que el modelo científico estudiado fuera para los estudiantes solo una representación canónica que han de aprender y memorizar, en vez de una herramienta para dar sentido a fenómenos naturales (Guy-Gaytán, Gouvea, Griesemer y Passmore 2019, Odden y Russ 2018).

También se ha evidenciado la importancia de considerar distintos modos de organización en el aula. La combinación de trabajo individual, en pequeño grupo y en gran grupo, aportó un contexto rico y estimulante que sirvió para integrar el trabajo reflexivo del estudiante, la discusión, y la aportación de ideas por parte del profesor. En particular, el trabajo en grupos se manifestó como una herramienta que potenciaba la reflexión individual, contribuyendo a que los estudiantes contrastaran, depuraran y mejoraran sus ideas (Velentzas y Halkia 2013).

Por otro lado, conviene destacar las aportaciones de los cambios introducidos en la SEA1, unas veces consecuencia de situaciones sobrevenidas que surgieron en el aula, y otras fruto de una revisión más pausada en función de los instrumentos de seguimiento. Nos referiremos solo a algunas que consideramos más relevantes.

Se ha de destacar el papel de las personificaciones, que se mostraron como herramientas excelentes para que los estudiantes protagonizaran in situ las analogías planteadas. En concreto, se emplearon dos, una de las cuales se introdujo premeditadamente como mejora en la SEA2, mientras la otra surgió sobre la marcha como oportunidad para aclarar las ideas que se manejaban. En ambos casos, los alumnos se implicaron notablemente en la actividad, y ello tuvo gran repercusión en las discusiones que se entablaron a raíz de ellas. Estos resultados refuerzan los aportados por la literatura que señalan la utilidad de las actividades encarnadas por estudiantes (embodiment), a través de las que hacen suyas las ideas manejadas mediante personificaciones. En efecto, como bien nos muestra Solbes y Tuzón (2014), la eficacia de esta 
estrategia radica en la activación física de un gran número de neuronas sensomotoras lo que propicia un aprendizaje más profundo que el que se logra con la mera observación (JohnsonGlenberg et al. 2012).

Igualmente, cabe reseñar la mejora introducida tras la simplificación de términos empleados por la profesora. Tales cambios hicieron más asequible las explicaciones aportadas y ofrecieron una oportunidad para que ellos mismos empoderaran un discurso propio más fluido y acorde con el de la docente. Según esto, las investigaciones de diseño pueden constituir un campo de prueba relevante para ajustar la terminología empleada en la enseñanza de las ciencias, como parte del proceso de transposición didáctica. De hecho, los estudios de micro-etnográfica de las interacciones comunicativas que ocurren en el aula, son considerados como una excelente estrategia metodológica para hacer visible y comprensible la relación entre lenguaje y conocimiento en el contexto de la enseñanza (Stubbs 1983).

Otro aspecto a considerar, y que señalamos en la sección de resultados, fue la conveniencia de simplificar el formato de los datos que los estudiantes han de manejar al trabajar con tablas o gráficos. En nuestro caso, por ejemplo, se sustituyeron tablas de mareas extraídas de una fuente formal, por una transcripción simplificada elaborada por la profesora. Ello ayudó a focalizar la atención de los alumnos en el sentido deseado, así como que extrajeran conclusiones apropiadas en relación al propósito de la actividad. Conviene recordar que Watson (2006) sugiere la importancia de las tablas y gráficos como instrumento que facilita la transición entre los datos y las inferencias estadísticas, si bien el tratamiento de datos por parte de los estudiantes no está exento de dificultades (Arteaga, Batanero, Cañadas y Contreras 2011).

Además, algunos cambios introducidos en los enunciados de las actividades parece que sirvieron para que los alumnos comprendieran mejor la tarea que tenían que realizar. Según se infiere de la implementación, los enunciados fueron más significativos cuando se estructuraron en torno a preguntas o problemas globales, que cuando se formularon mediante múltiples apartados, que lejos de orientar el trabajo de los estudiantes, fragmentaban el significado de la actividad y perdían de vista su intencionalidad. Esta conclusión es coherente con el marco de la enseñanza mediante modelización, ya que los modelos se construyen como representaciones articuladas que dan respuesta a un problema (Gobert y Bucley 2000; Reiner y Burko 2003). En este sentido, la fragmentación de los enunciados podría contribuir poco, a la construcción de un escenario global que invite a evocar un modelo.

Finalmente, se detecta que el orden de las actividades puede suponer un factor importante a la hora de establecer un hilo conductor en la secuencia. De este modo, los cambios en el orden de las actividades, parece que contribuyeron a una secuenciación más gradual y significativa de algunas de las partes del diseño didáctico implementado.

\section{Agradecimientos}

Financiado por: FEDER/Ministerio de Ciencia, Innovación y Universidades-Agencia Estatal de Investigación/_Proyecto EDU2017-82518-P.

\section{Referencias}

Acher A. (2014) Cómo facilitar la modelización científica en el aula. Tecné, Episteme y Didaxis: TED 36, 63-75. http://dx.doi.org/10.17227/01213814.36ted63.75 
Armario M., Jiménez-Tenorio N., Oliva J.M. (2019) El fenómeno de las mareas y su explicación. Una propuesta de progresión para su enseñanza. Alambique Didáctica de las Ciencias Experimentales 95, 31-37.

Armario M., Jiménez-Tenorio, N., Oliva, J.M. (2021) Secuencia didáctica sobre el fenómeno de las mareas. Rodin. Repositorio Oficial de la Universidad de Cádiz. http://hdl.handle.net/10498/25254

Armario M., Oliva J.M., Jiménez-Tenorio, N. (2021) Spanish preservice primary school teachers' understanding of the tides phenomenon. International Journal of Science and Mathematics Education (en prensa).

Arteaga P., Batanero C., Cañadas G., Contreras J.M. (2011) Las tablas y gráficos estadísticos como objetos culturales. Números: Revista de Didáctica de las Matemáticas 76, 56-67.

Ballantyne R. (2004) Young student's conceptions of the marine environment and their role in the development of aquaria exhibits. GeoJounal 60, 159-163. https://doi.org/10.1023/B:GEJO.0000033579.19277.ff

Clement J. (2000) Model based learning as a key research area for science education. International Journal of Science Education 22 (9), 1041-1053. https://doi.org/10.1080/095006900416901

Camelo F.J., Rodríguez S.J., Santiesteban S.N. (2007) Ideas previas. Un constructo indispensable en el diseño de situaciones en el aula: un ejemplo en ciencias. Horizonte Pedagógico 9 (1), 89-100.

Corrochano D., Gómez-Gonçalves A., Sevilla J., Pampín-García S. (2017) Ideas de estudiantes de instituto y de universidad acerca del significado y el origen de las mareas. Revista Eureka sobre Enseñanza y Divulgación de las Ciencias 14 (2), 353-366. http:/ /dx.doi.org/10.25267/Rev_Eureka_ensen_divulg_cienc.2017.v14.i2.05

Delgado-Serrano R., Cubilla K. (2012) La necesidad de investigar la comprensión de conceptos básicos, de Astronomía y Ciencias en general, en pre-media y media. Latin American and Caribbean Conference for Engineering and Technology 10, 23-27.

Galili I., Lehavi Y. (2003) The importance of weightlessness and tides in teaching gravitation. American Journal of Physics 71 (11), 1127-1135. http://dx.doi.org/10.1119/1.1607336

Galperin D., Raviolo, A. (2014) Sistemas de referencia en la enseñanza de la Astronomía. Un análisis a partir de una revisión bibliográfica. Latin American Journal of Physics Education, 8, 136-148.

Garrido A. (2016) Modelitració i models en la formació inicial de mestres de primària des de la perspectiva de la pràctica científica (Tesis). Universidad autónoma de Barcelona, Bellaterra.

Gilbert J.K., Justi R. (2016) Modelling-based teaching in science education. Basel: Springer.

Gobert J.D., Buckley B.C. (2000) Introduction to model-based teaching and learning in science education. International Journal of Science Education 22 (9), 891-894. https://doi.org/10.1080/095006900416839

Guy-Gaytán C., Gouvea J.S., Griesemer C., Passmore C. (2019) Tensions between learning models and engaging in modeling. Exploring implications for science classrooms. Science \& Education 28, 843-864. https://doi.org/10.1007/s11191-019-00064-y

Halloun I. (2007) Mediated modeling in science education. Science \& Education 16, 653-697. https://doi.org/10.1007/s11191-006-9004-3 
Hartel H. (2000) The tides: a neglected topic. Physics Education 35 (1), 40-45.

Jorba J., Sanmartí N. (1996) El desarrollo de las habilidades cognitivolingüisticas en la enseñanza de las ciencias. Mimeo: Barcelona.

Johnson-Glenberg M., Lindgren R., Koziupa T., Bolling A., Nagendran A., Birchfield D., Cruse J. (2012) Serious games in embodied mixed reality learning environments. Games Learning and Society Conference 8, 8.

Justi R., Gilbert J.K. (2002) Modelling teachers' views on the nature of modelling, and implications for the education of modellers. International Journal of Science Education 24 (4), 369-387. https://doi.org/10.1080/09500690110110142

Kallai A.Y., Reiner M. (2010) The source of misconceptions in physics: when event-related potential components N400 and P600 disagree. Front. Neurosci. Conference Abstract: EARLI SIG22 - Neuroscience and Education. bttps:/ / doi.org/10.3389/conf.fnins.2010.11.00065

López V. (2014) Aprende: las mareas. Programa 'Con-ciencia' de Canal Sur TV, Recuperado de: https://www.youtube.com/watch?v=YCQB6HDBuyE

Molina M., Castro E., Molina J.L., Castro E. (2011) Un acercamiento a la investigación de diseño a través de los experimentos de enseñanza. Enseñanza de las Ciencias 29 (1), 075088.

Nicolaou C.T., Constantinou C.P. (2014) Assessment of the modeling competence: A systematic review and synthesis of empirical research. Educational Research Review 13, 5273. https://doi.org/10.1016/j.edurev.2014.10.001

Norsen T., Dreese M., West C. (2017) The gravitational self-interaction of the Earth's tidal bulge. American Journal of Physics 85 (9), 663-669. https://doi.org/10.1119/1.4985124

Odden T.O.B., Russ R.S. (2018) Defining sensemaking: bringing clarity to a fragmented theoretical construct. Science Education 103 (1), 187-205. https://doi.org/10.1002/sce.21452

Oh J.Y. (2014) Understanding the alternative conceptions of pre-service secondary science teachers about tidal phenomena based on Toulmin's argumentation. International Journal of Science and Mathematics Education 12 (2), 353-370. https://doi.org/10.1007/s10763013-9403-2

Oh S.P., Oh S.J. (2011) What teachers of science need to know about models: an overview. International Journal of Science Education 33 (8), 1109-1130. https://doi.org/10.1080/09500693.2010.502191

Oliva J.M. (2019) Distintas acepciones para la idea de modelización en la enseñanza de las ciencias. Enseñanza de las Ciencias 37 (2), 5-24.

Pozo J.I., Pérez M.P., Sanz A., Limón M. (1992) Las ideas de los alumnos sobre la Ciencia como teorías implícitas. Infancia y Aprendizaje 57, 3-22.

Railsback L.N. (1991) A model for teaching the dynamical theory of tides. Journal of Geological Education 39 (1), 15-18. https:/ / doi.org/10.5408/0022-1368-39.1.15

Reiner, M. y Gilbert, J. (2000). Epistemological resources for thought experimentation in science learning. International Journal of Science Education, 22(5), 489-506. https://doi.org/10.1080/095006900289741 
Reiner M., Burko L. (2003) On the limitations of thought experiments in physics and the consequences for physics education. Science and Education 12, 385-358. https://doi.org/10.1023/A:1024438726685

Seel N.M. (2017) Model-based learning: A synthesis of theory and research. Educational Technology Research and Development 65 (4), 931-966. https://doi.org/10.1002/tea.2031110.1007/s11423-016-9507-9

Simanek D.E. (2015) Tidal Misconceptions. Recovered from: https://www.lockhaven.edu/ dsimanek/scenario/tides.htm

Solbes J., Palomar R. (2011) ¿Por qué resulta tan difícil la comprensión de la Astronomía a los estudiantes? Didáctica de las ciencias experimentales y sociales 25, 187-211.

Solbes J., Tuzón P. (2014) Indagación y modelización del núcleo atómico y sus interacciones. Alambique: Didáctica de las ciencias experimentales 78, 34-12.

Stubbs M. (1983) Language, schools and classrooms. London: Metheun.

Ucar S., Trundle K.C., Krissek L. (2011) Inquiry-based instruction with archived, online data: An intervention study with preservice teachers. Research in Science Education 41 (2), 261282. https://doi.org/10.1007/s11165-009-9164-7

Velentzas A., Halkia K. (2013) The use of thought experiments in teaching physics to upper secondary-level students: Two examples from the theory of relativity. International Journal of Science Education 35 (18), 3026-3049. https://doi.org/10.1080/09500693.2012.682182

Viiri J. (2000) Students' understanding of tides. Physics Education 35, 105. https://doi.org/10.1088/0031-9120/35/2/305

Viiri J., Saari H. (2004) Research-based teaching unit on the tides. International Journal of Science Education 26, 463-481. https://doi.org/10.1080/0950069032000072791

Watson J.M. (2006) Statistical literacy at school: growth and goals. Mahwah, NJ: Lawrence Erlbaum Associates. 
Anexo I. Cuestionario empleado en el Pretest y en el Postest del estudio.

\section{Datos iniciales}

Nombre y apellidos:

Edad:

Sexo: mujer/hombre:

Curso:

Centro:

Localidad:

¿Con qué frecuencia vas a la playa o tienes contacto con el mar?

¿Tienes alguna afición relacionada con el mar? (Ej: pescar, mariscar, surf...) Indica cuáles. ¿Y tu familia?

Cuando pasas un día completo en la playa vas observando cambios a tu alrededor. ¿Podrías mencionar algunos que te parezcan relevantes desde el punto de vista científico?

¿Te gustaría saber más sobre estos cambios?

\section{Cuestionario sobre el fenómeno de las mareas}

1. ¿Qué son las mareas?

2. ¿Cada cuánto tiempo se producen?

3. ¿Las subidas y bajadas de marea son iguales en todas las partes del mundo?

4. ¿Crees que tienen las mareas algo que ver con otros fenómenos que conozcas? Si es así, indica con cuáles y explica por qué lo crees.

5. ¿Por qué se producen las mareas? Utiliza tus palabras y realiza un dibujo que te ayude en la explicación.

6. Si en el punto de la Tierra señalado con un círculo la marea es alta, ¿Cómo será el nivel del mar en el punto opuesto de la misma, justo donde aparece el triángulo? Explícalo con tus palabras y realiza un dibujo.

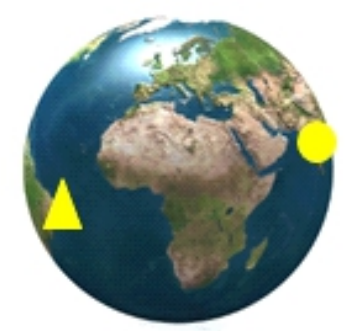

7. Un pescador llega a la playa a las 20:00 y observa que el nivel del mar está bajando. Si pasa toda la noche allí, ¿Cuál será el nivel del mar a las 8:00? Explícalo

8. ¿Habría marea en la Luna si tuviera océanos? Explícalo 